\title{
Analysis of Slotting Models for the Calculation of No-Load Rotor Losses in PM Machines
}

\author{
J. R. Anglada, Student Member, IEEE, S. M. Sharkh, Senior Member, IEEE, and A. A. Qazalbash
}

\begin{abstract}
The magnetic flux density distribution in the airgap of electric machines is essential for accurate prediction of no-load eddy-current power losses. The effect of slotting can be modelled using a simplified single-slot model or a complete multiple-slots model. Until now there has not been a clear and justified criterion to choose between the two models. In this paper we propose a criterion based on the conformal transformations used to calculate the magnetic field distribution of single-slot and multiple-slots models. The computational implementation of both methods produced a graph that clearly shows which method to use as a function of the normalised geometrical parameters of the machine. The paper presents a case study of a high speed machine whose proportions fall within the multiple-slot model range, according to the criterion proposed. It is shown that using a single slot model in this case results in significant errors in the estimation of rotor losses. Better agreement with FEA results is achieved when a multipleslot model is used.
\end{abstract}

Index Terms-Analytical models, permanent magnet machines, conformal mapping, rotor eddy-current power loss calculation.

\section{INTRODUCTION}

A CCURATE calculation of the magnetic field in the airgap of electric machines is essential when calculating rotor losses, cogging torque and similar quantities that strongly depend on the harmonic content of the magnetic field distribution [1]-[5]. High accuracy is crucial in the case of rotor losses as small errors can result in designing a machine that will run too hot if the losses are underestimated. Overestimating the losses could result in a decision to abandon a good design variant and opting for expensive solutions such as magnet segmentation or increasing the air-gap length or the magnet and sleeve thickness.

The methods used to calculate the magnetic field in the air-gap of electric machines can be classified into two main groups: numerical methods or analytical methods. Numerical methods, like Finite Element Analysis (FEA), are extremely useful tools because they are versatile and accurate. However, the computation time tends to be high and in general it is difficult to gain an insight from the these solutions unless

This work was supported by EPSRC and by TSL Technology.

J. R. Anglada and S. M. Sharkh are with the Mechatronics Research Group, Engineering Sciences, Faculty of Engineering and the Environment, University of Southampton, Highfield Campus, Southampton SO17 1BJ, United Kingdom (e-mail: j.renedo-anglada@soton.ac.uk; suleiman@soton.ac.uk).

A. A. Qazalbash is with Emerson Control Techniques Dynamics Ltd, UK (email: arfakhshand.qazalbash@hotmail.com). several geometries are analysed; some numerical solvers provide tools for parametric analysis for this purpose [6], [7]. Numerical methods remain very useful tools for the validation and the refinement of the final design.

On the other hand, analytical methods are still very useful tools for initial design and optimisation based on the insight obtained. Carter pioneered the use of conformal mapping, which transforms a slotted geometry of the air-gap into a slotless one in which the field could be calculated. He defined a coefficient to quantify the effect of slotting on the mean value of the magnetic field waveform [8]. However, the transformation from the slotted geometry into the slotless one is a Schwarz-Christoffel (SC) transformation that does not have an explicit expression for complicated domains [9] which made the practical application of this method difficult. Gibbs [10] extended Carter's work by developing two different methods also based on conformal mapping: one is a simplification considering infinitely wide teeth and the other one takes into account the effect of the neighbouring slots. Freeman [11] applied Gibbs' methods to a range of practical geometries and expressed the magnetic field distribution as a Fourier series. Even though Gibbs' method can be solved, the SC transformation of the multiple slots model is so complicated that in most of the cases the single slot solution is preferred [3]-[5], [12]-[14].

The problem of neglecting the effect of the neighbouring slots, i. e., using a single-slot model, is that when the teeth are narrow and the air-gap is large the waveform obtained may not be of sufficient accuracy. This is commonly the case in PM machines which have large effective air-gaps. In [3]-[5] for example, the machines analysed have very large effective air-gaps with thick magnets and sleeves. In these machines a strong influence from the adjacent slots is expected and a multiple-slot model would be needed. But in the literature only Freeman suggested a criterion to choose between single-slot and the multiple-slots models, but without any clear justification behind this assertion [11].

The aim of this paper is to understand the limitations of the single-slot model and develop a criterion that can be used to determine when it is valid to apply it for the calculation of rotor eddy-current power loss calculation. The methodology chosen for the analysis is Gibbs' single-slot and multipleslots methods because they are completely analytical and the assumptions of both models are exactly the same except for the width of the teeth. For tooth widths that are higher than a certain value the influence of the adjacent slots can be neglected and the single-slot model is valid. For smaller tooth 
widths there is an interaction between the neighbouring slots and the single-slot model will be inaccurate. In theory the multiple-slots model should always provide correct results but in practice for geometries with very large tooth widths the transformation is ill defined making the numerical solvers fail to find a solution. In these cases the single-slot model should be used.

In this paper we propose a clear and explicit criterion for choosing between single-slot and multiple-slots methods. This criterion is based on the properties of the conformal transformations proposed by Gibbs [10]. The graphical representation of the limits of application of each of the methodologies allows us to know immediately which model to use in each case.

The paper starts with a summary of methodology to calculate rotor eddy-current power loss calculation using a current sheet model. Then, Gibbs' methodologies for the single-slot and multiple-slots geometries are presented briefly. Next, section IV shows how the limits of application of each of the methodologies are obtained. The results are presented in section IV-C; Fig. 5 covers the typical geometries and clearly states which model should be used in each case. Also, an example is presented to illustrate the significant errors introduced when neglecting the effect of the neighbouring slots in the calculation of rotor losses in a PM machine. Finally, the conclusions are presented in section VI.

\section{Calculation of No-Load Rotor Eddy-CurRent POWER LOSS}

No-load rotor eddy-current power loss is calculated using a cylindrical multilayer model in which each asynchronous harmonic is represented by a current sheet at the stator bore of a slotless configuration of the machine; this is represented schematically for 4 layers in Fig. 1. The nonsegmented magnet is modelled as a conducting region with no magnetization.

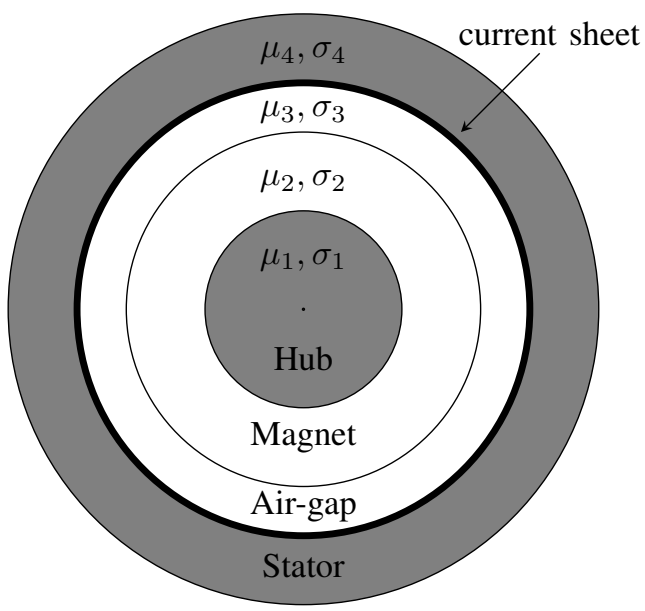

Fig. 1. Cylindrical model of the PM machine with the corresponding current sheet at the stator bore.

The current sheet density of an asynchronous harmonic of space order $q$ and time order $k$ can be expressed as

$$
J_{q k}=\hat{J}_{q k} \cos (q \theta+k \omega t)=\operatorname{Re}\left(\hat{J}_{q k} e^{j q \theta} e^{j k \omega t}\right) .
$$

The amplitude of the current sheet that corresponds to each asynchronous harmonic, $\hat{J}_{q k}$, is effectively set to produce the same normal flux density on the surface of the magnet $\hat{B}_{q k}$ [3]-[5]. In practice, the problem is solved by setting $\hat{J}_{q k}=1$ and calculating the corresponding losses $P_{q k 1}$ from the solution of the diffusion equation in the current sheet model. Also, the Laplace equation (no eddy currents) is solved to find the corresponding $\hat{B}_{q k 1}$ when $\hat{J}_{q k}=1$. Finally, the actual losses for a given $\hat{B}_{q k}$ (obtained from harmonic analysis using conformal mapping in this case) are calculated as

$$
P_{q k}=\left(\frac{\hat{B}_{q k}}{\hat{B}_{q k 1}}\right)^{2} P_{q k 1} .
$$

In this paper we study the no-load rotor loss of a nonsalient PM machine. From the rotor reference frame the slots change position with time and this variation of permeance produces a variation of the magnetic field seen by the rotor which induces eddy currents [1], [2]. The calculation of the asynchronous harmonics due to tooth permeance variation is done by calculating the magnetic field distribution in different rotor positions spanning one pole-pitch as shown in Fig. 2.

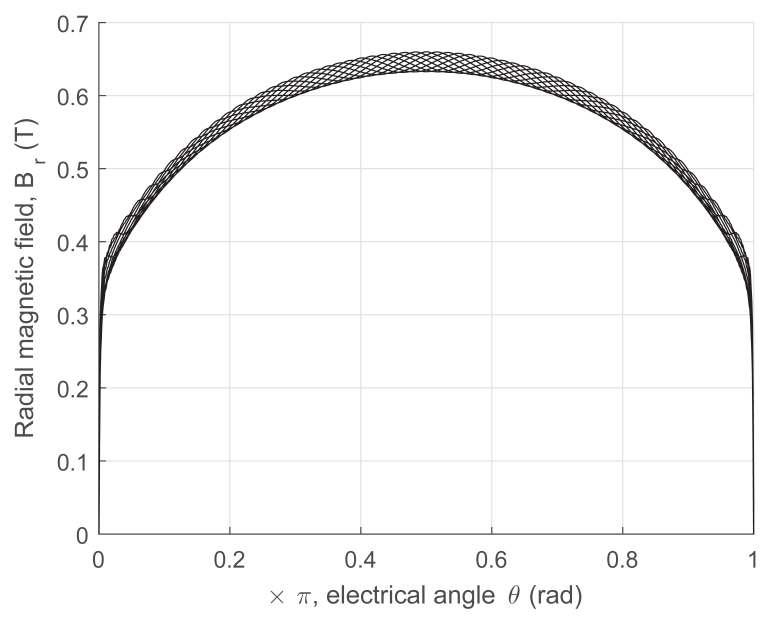

Fig. 2. Radial component of the magnetic field on the surface of the magnet for several rotor positions.

The amplitude of the space and time harmonics are calculated using a two-dimensional discrete Fourier transform on a matrix of the waveforms of the magnetic field at several rotor positions. In this case one dimension corresponds to time and the other one to space.

In this paper the waveforms of the magnetic field distribution are obtained analytically using a complex permeance (CP) function and from magnetostatic FEA to compare the amplitude of the space and time asynchronous harmonics.

\section{Slotting Models}

Conformal mapping uses analytic complex functions to transform one domain into another while preserving the 
angles [10]. Using conformal mapping we can transform a domain - a rectangular polygon in this case - into a new one in which the solution is known. If we call the original domain the $z$-plane and the new one the $w$-plane we can find the relationship between their magnetic fields. For an arbitrary analytic complex function $w=f(z)$ the expression that relates the magnetic field in both planes [13] is

$$
B_{z}=B_{w}\left(\frac{d w}{d z}\right)^{*}=B_{w}\left(f^{\prime}(z)\right)^{*},
$$

where the asterisk denotes the complex conjugate. The Schwarz-Christoffel (SC) transformation is commonly used to find the suitable function $f(z)$. By definition an SC transformation can map the interior of an arbitrary polygon in the upper half of the complex plane [9].

The following methods based on [10], [11] show how to obtain the magnetic field distribution in the air-gap of electric machines with a toothed member. The air-gap has a magnetic permeability of $\mu_{0}$ and the iron permeability is assumed to be infinity.

\section{A. Single-Slot Model}

Fig. 3 shows the geometry considered for a single slot surrounded by infinitely wide teeth that we will call the $z$ plane. The field is produced by a magneto-motive force of $V$ between the toothed member and the pole-face.

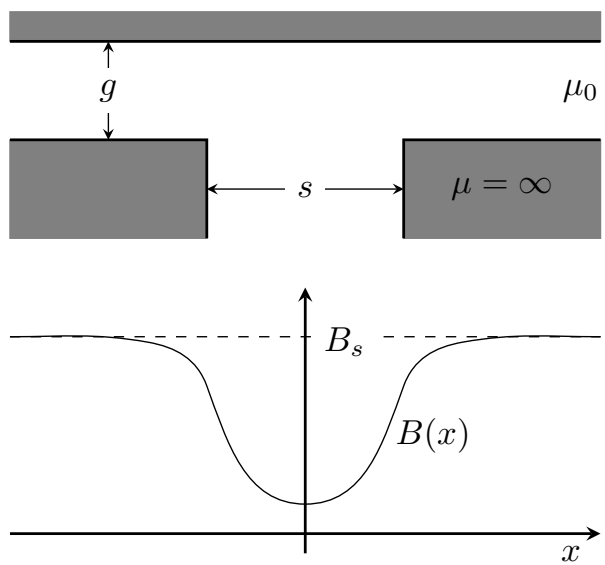

Fig. 3. Geometry of a single slot and the corresponding air-gap magnetic flux density waveform.

The magnetic flux density $B$ on the pole-face as a function of the intermediate variable $w$ can be shown to be given by

$$
B(w)=\frac{w-1}{(w-a)^{\frac{1}{2}}(w-b)^{\frac{1}{2}}} B_{s},
$$

with $a=1 / b$ and $b$ is obtained from the equation:

$$
\frac{b-1}{\sqrt{b}}=\frac{s}{g} .
$$

$B_{s}$ is the value of the magnetic flux density if there were no slots:

$$
B_{s}=\frac{\mu_{0} V}{g} .
$$

The distance along the pole-face,

$$
\begin{aligned}
x=\frac{g}{\pi}\left\{-\log \left|\frac{1+p}{1-p}\right|\right. & +\log \left|\frac{b+p}{b-p}\right| \\
& \left.+\frac{2(b-1)}{\sqrt{b}} \tan ^{-1} \frac{p}{\sqrt{b}}\right\}-\frac{s}{2},
\end{aligned}
$$

with the parameter $p$ given by the intermediate variable $w$ :

$$
p^{2}=\frac{b-w}{a-w} .
$$

To obtain the magnetic field distribution $B$ and $x$ are evaluated as a function of $w$ for values from -1 to 0 .

\section{B. Multiple-Slots Model}

The objective is to transform the geometry shown in Fig. 4 into a rectangle to solve the Laplace equation with the same assumptions as in the previous section. The main difference between this method and the one that considers a single-slot is that there is not an explicit equation for the transformation. This is why it is necessary to operate with the intermediate variables $\alpha$ and $k$. The parameters $\alpha$ and $k$ are obtained by
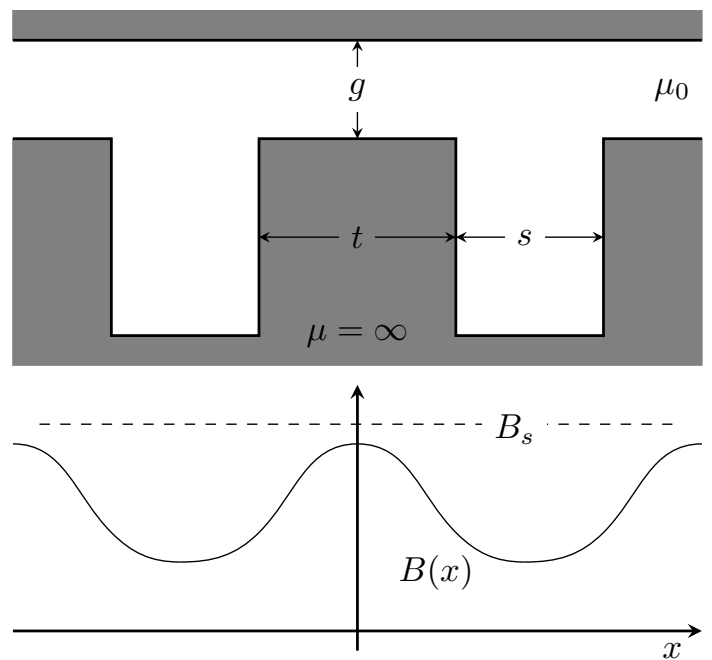

Fig. 4. Geometry of a machine with multiple slots and the corresponding magnetic flux density waveform.

solving the following system of non-linear equations:

$$
\begin{gathered}
\frac{g}{s}=\frac{\mathrm{K}(k)}{\pi}\left\{\frac{\operatorname{sn}(\alpha, k) \operatorname{dn}(\alpha, k)}{\operatorname{cn}(\alpha, k)}-\mathrm{Z}(\alpha, k)\right\}, \\
\frac{t}{s}=\frac{2 \mathrm{~K}\left(k^{\prime}\right)}{\pi}\left\{\frac{\operatorname{sn}(\alpha, k) \operatorname{dn}(\alpha, k)}{\operatorname{cn}(\alpha, k)}-\mathrm{Z}(\alpha, k)\right\}-\frac{\alpha}{\mathrm{K}(k)},
\end{gathered}
$$

with

$$
k^{\prime}=\sqrt{1-k^{2}}
$$

where $\mathrm{K}(k)$ is the complete elliptic integral of the first kind. The functions $\operatorname{sn}(\alpha, k), \operatorname{cn}(\alpha, k)$ and $\operatorname{dn}(\alpha, k)$ are the Jacobi trigonometric functions defined as inverse elliptic integrals. Finally, $\mathrm{Z}(\alpha, k)$ is the Jacobi Zeta function defined as a function of the elliptic integrals [15]. 
The expression for the magnetic flux density as a function of the intermediate variable $v$ is:

$$
B(v)=\frac{\left(1+k_{1}^{2} v^{2}\right)^{\frac{1}{2}}}{\left(1+k^{2} v^{2}\right)^{\frac{1}{2}}} B_{\max }
$$

where

$$
B_{\max }=\frac{\pi g}{s \mathrm{~K}\left(k_{1}\right)} \frac{\operatorname{cn}(\alpha, k)}{\operatorname{sn}(\alpha, k) \operatorname{dn}(\alpha, k)} B_{s},
$$

with $k_{1}=k \operatorname{sn}(\alpha, k)$.

The distance along the pole-face,

$$
\begin{aligned}
& x(v)=\frac{s}{\pi}\left[\left\{\frac{\operatorname{sn}(\alpha, k) \mathrm{dn}(\alpha, k)}{\operatorname{cn}(\alpha, k)}-\mathrm{Z}(\alpha, k)\right\} \beta\right. \\
& \left.+\tan ^{-1} \frac{-2 \sum_{1}^{\infty}(-1)^{m} q^{m^{2}} \sin \frac{\pi m \alpha}{\mathrm{K}(k)} \sinh \frac{\pi m \beta}{\mathrm{K}(k)}}{1+2 \sum_{1}^{\infty}(-1)^{m} q^{m^{2}} \cos \frac{\pi m \alpha}{\mathrm{K}(k)} \cosh \frac{\pi m \beta}{\mathrm{K}(k)}}\right],
\end{aligned}
$$

with

$$
\beta=\mathrm{F}\left(\frac{v}{\left(1+v^{2}\right)^{\frac{1}{2}}}, k^{\prime}\right),
$$

where $\mathrm{F}(\phi, k)$ is the incomplete elliptic integral of the first kind and $q$ is called the nome, $q=e^{-\frac{\pi \mathrm{K}\left(k^{\prime}\right)}{\mathrm{K}(k)}}$.

To obtain the flux density distribution $B$ and $x$ are evaluated as a function of $v$ for values from 0 to $\infty$ in a similar way as in the previous method.

\section{Limits OF AppliCATION}

\section{A. Practical Limit}

When the teeth are wide enough it was noted by Gibbs and Freeman that the maximum value of the flux density, $B_{\max }$, is almost equal to $B_{s}$. This suggests that the effect of neighbouring slots on the field distribution in the vicinity of a slot is negligible.

We can define the following indicator to study if the single-slot model is going to give almost the same answer as the multiple-slots model for a particular geometry:

$$
r_{p}=\frac{B_{\max }}{B_{s}}=\frac{\pi g}{s \mathrm{~K}\left(k_{1}\right)} \frac{\operatorname{cn}(\alpha, k)}{\operatorname{sn}(\alpha, k) \operatorname{dn}(\alpha, k)},
$$

where $B_{\max }$ is obtained from (13). With this indicator for any geometry (a given $t / s$ and $g / s$ ) we can estimate immediately if both models give a similar answer. If the value of $r_{p}$ is close to be 1 it means that the interaction between adjacent slots is negligible and a single-slot model can be used. If it is significantly smaller than 1 then a multiple-slot model is needed.

\section{B. Numerical Limit}

This section shows the range of the geometrical variables within which the multiple-slots model is valid. Theoretically, according to the definition of the Schwarz-Christoffel transformation the geometry of Fig. 4 can always be mapped into a rectangle. However, in practice when the ratio of the tooth width $t$ and the air-gap length $g$ is large, i.e., the teeth are very wide, the numerical solution of (9) and (10) becomes impossible.

Let us define the right hand side of (9) as $\mathrm{F}_{\mathrm{g}}(\alpha, k)$ and the right hand side of $(10)$ as $\mathrm{F}_{\mathrm{t}}(\alpha, k)$ :

$$
\begin{gathered}
\frac{g}{s}=\mathrm{F}_{\mathrm{g}}(\alpha, k), \\
\frac{t}{s}=\mathrm{F}_{\mathrm{t}}(\alpha, k) .
\end{gathered}
$$

Considering a particular value of $\frac{g}{s}=K$, a curve $\Gamma_{K}$ of all the points $(\alpha, k)$ that satisfy this equation can be defined as the following:

$$
\left(\alpha_{i}, k_{i}\right) \in \Gamma_{K} \Leftrightarrow \mathrm{F}_{\mathrm{g}}\left(\alpha_{i}, k_{i}\right)=K .
$$

Of all the points in $\Gamma_{K}$ there is only one point $\left(\alpha_{o p t}, k_{\text {opt }}\right)$ that satisfies:

$$
\mathrm{F}_{\mathrm{t}}\left(\alpha_{\text {opt }}, k_{\text {opt }}\right)=\frac{t}{s} .
$$

To know the limits of application of the multiple-slots methodology we need to find the maximum tooth width within which the numerical solver can provide a solution. To find the maximum value of $\frac{t}{s}$ for a particular value of $\frac{g}{s}$ an algorithm was implemented in MATLAB. The algorithm is divided in three stages:

(a) Choose a value of $\frac{g}{s}=\mathrm{F}_{\mathrm{g}}(\alpha, k)=K$.

(b) Obtain the curve (family of points) $\Gamma_{K}$.

(c) Calculate the point $\left(\alpha_{o p t}, k_{o p t}\right)$ that maximizes the function $\mathrm{F}_{\mathrm{t}}(\alpha, k)$ and evaluate $\left.\frac{t}{s}\right|_{\max }$.

The value of $\left.\frac{t}{s}\right|_{\max }$ will depend on the numerical precision of the software.

For this case as the value of $\frac{t}{s}$ increases $k^{\prime}$-see equation (11) — tends to be close to zero. This results in $k$ being close to 1 and the elliptic integral of the first kind has the following property:

$$
\lim _{k^{\prime} \rightarrow 0} \mathrm{~K}\left(k^{\prime}\right)=\infty \Rightarrow \lim _{k \rightarrow 1} \mathrm{~F}_{\mathrm{t}}(\alpha, k)=\infty .
$$

For this reason the numerical limit in which the multiple slots method has a solution will depend on the numerical precision of the software; for the case of MATLAB the minimum value of $k^{\prime}$ in which $\mathrm{K}\left(k^{\prime}\right)$ is not infinite is $k^{\prime}=10^{-8}$.

\section{Representation of the Limits}

This section presents the results obtained with MATLAB after implementing the algorithms to calculate the practical and numerical limits. To obtain the limits the previous methodology was applied for a range of values of $\frac{g}{s}$ to calculate the corresponding $\left.\frac{t}{s}\right|_{\max }$.

Freeman [11] proposed the following criterion: if $t / g>$ 3.3 the single-slot model should be used and if $t / g<3.3$ the multiple-slots model should be used. This condition can also be expressed using the normalised parameters $\frac{t}{s}$ and $\frac{g}{s}$ :

$$
\frac{t}{s} \lessgtr 3.3 \frac{g}{s} \text {. }
$$




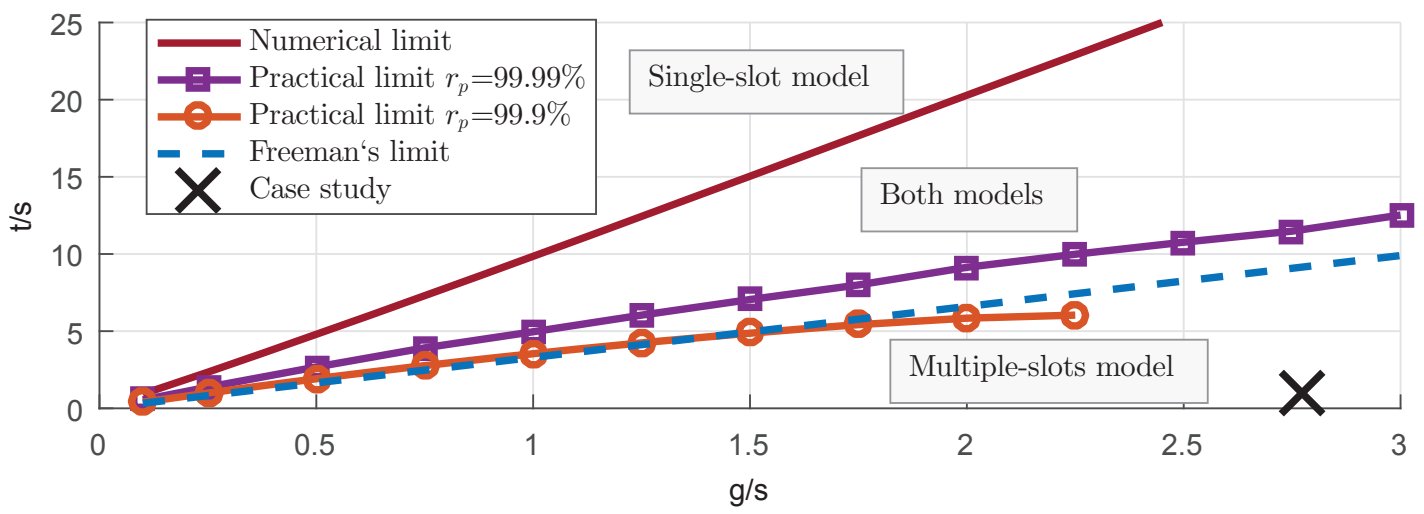

Fig. 5. Numerical and practical limits of the two methodologies as a function of the geometric variables. Also, representation of Freeman's limit and the position of the machine analysed in section $\mathrm{V}$.

Fig. 5 shows the limit proposed by Freeman, the numerical limit and the practical limit for 3 different values of $r_{p}$ as a function of the normalised variables $\frac{t}{s}$ and $\frac{g}{s}$. The figure can be divided in three different regions. In the region above the solid red line the single-slot model should always be used because the multiple-slots model will fail as it was noted in section IV-B. Below the orange line with the circular markers ignoring the effect of the neighbouring slots can produce significant errors because the magnetic field in the middle of the teeth does not reach $B_{s}$ as it was described in section IV-A (here the minimum value of $r_{p}$ was considered to be the $99.9 \%)$. Between these two lines both models can be used in the sense that they will give similar answers. However, above the magenta line with square markers that is $r_{p}$ of $99.99 \%$ the solution of both methods will be almost identical and $k^{\prime} \rightarrow 0$. Freeman's limit in Fig. 5 is the dashed blue line. It is almost the same as the practical limit with $r_{p}$ of $99.9 \%$.

\section{CAse Study}

Fig. 6 shows a quarter of the cross-section of a high speed PM generator with a non-conductive rotor sleeve to hold the magnets, making the effective air-gap even larger [3]. The parameters of this machine are shown in table I.

TABLE I

PaRAMETERS OF THE MACHINE

\begin{tabular}{lcc}
\hline Quantity & Symbol & Value \\
\hline Number of poles & $2 p$ & 4 \\
Number of slots & $Q_{s}$ & 24 \\
Core length & $L$ & $125 \mathrm{~mm}$ \\
Rotor radius & $R_{1}$ & $21.6 \mathrm{~mm}$ \\
Magnet outer radius & $R_{2}$ & $27.1 \mathrm{~mm}$ \\
Stator radius & $R_{3}$ & $31 \mathrm{~mm}$ \\
Magnet thickness & $h_{m}$ & $5.5 \mathrm{~mm}$ \\
Sleeve thickness & $t_{s l}$ & $2 \mathrm{~mm}$ \\
Clearance gap & $h_{g}$ & $1.9 \mathrm{~mm}$ \\
Slot opening & $b_{o}$ & $3 \mathrm{~mm}$ \\
Rotor hub permeability & $\mu_{r}$ & 750 \\
Rotor hub conductivity & $\sigma_{r}$ & $6.7 \cdot 10^{6} \mathrm{~S} / \mathrm{m}$ \\
Magnet conductivity & $\sigma_{m}$ & $0.77 \cdot 10^{6} \mathrm{~S} / \mathrm{m}$ \\
Magnet material & - & $\mathrm{NdFeB}$ \\
Magnet remanence & $B_{r}$ & $1.07 \mathrm{~T}$ \\
Magnet coercivity & $H_{c}$ & $851 \mathrm{kA} / \mathrm{m}$ \\
\hline
\end{tabular}

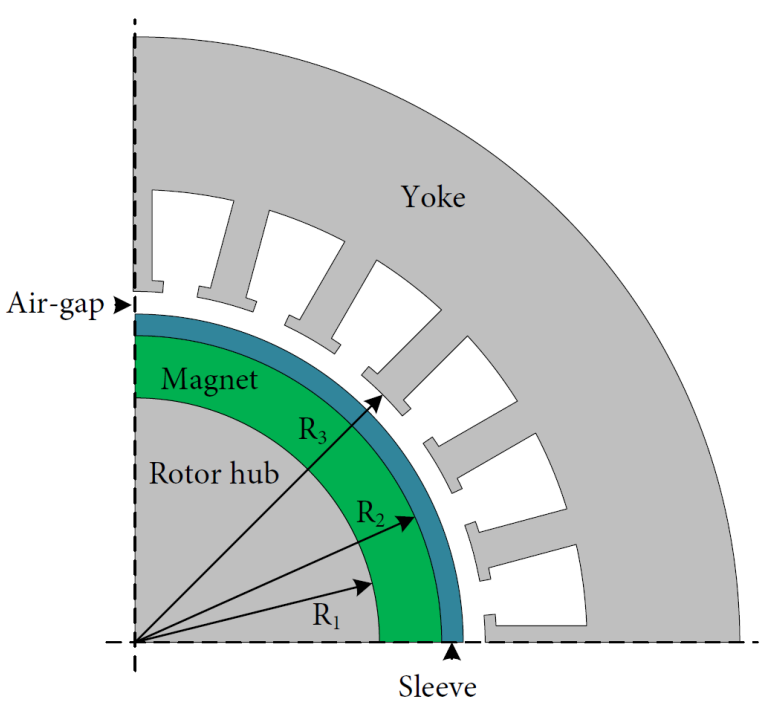

Fig. 6. Quarter model of the PM synchronous generator under study.

Taking into account that the permeability of the magnets and the sleeve is close to $\mu_{0}$ the effective air-gap length:

$$
g=h_{m}+t_{s l}+h_{g}=9.4 \mathrm{~mm} .
$$

In the developed model of the machine $t=3.492 \mathrm{~mm}$ and $s=3.394 \mathrm{~mm}$, therefore

$$
\begin{aligned}
\frac{g}{s} & \approx 2.770, \\
\frac{t}{s} & \approx 1.029 .
\end{aligned}
$$

The position of this machine in Fig. 5 is shown with a black cross. Clearly the machine is in the region where only the multiple-slots model should be used. To study the limitations of the single-slot model the CP function is obtained for a multiple-slots and single-slot configuration for comparison. Both methodologies include a first conformal transformation to model the effect of curvature [16].

The no-load magnetic field distribution in the air-gap of the slotless configuration using the rotor's reference frame 
can be expressed using complex number notation as

$$
\begin{aligned}
B_{s l}(\theta, r)=\sum_{n=1,3,5}^{\infty} K_{n}(r) & \cos (n p \theta) \\
& +j \sum_{n=1,3,5}^{\infty} D_{n}(r) \sin (n p \theta),
\end{aligned}
$$

where the coefficients $K_{n}(r)$ and $D_{n}(r)$ are calculated according to [17] and $j=\sqrt{-1}$ is the pure imaginary part. The CP function (both for the multiple-slots and single-slot models) using the rotor's reference frame is

$$
\begin{aligned}
\lambda(\theta, r, t)= & \lambda_{a 0}+\sum_{m=1,2,3}^{\infty} \lambda_{a m}(r) \cos \left(m Q_{s}(\theta-\omega t)\right) \\
& +j \sum_{m=1,2,3}^{\infty} \lambda_{b m}(r) \sin \left(m Q_{s}(\theta-\omega t)\right),
\end{aligned}
$$

where the coefficients $\lambda_{a 0}, \lambda_{a m}(r)$ and $\lambda_{b m}(r)$ are calculated using conformal mapping. Therefore, according to [13] the magnetic field distribution of the slotted geometry is

$$
B(\theta, r, t)=B_{s l}(\theta, r) \cdot \lambda^{*}(\theta, r, t) .
$$

The radial and tangential components of the CP function on the surface of the magnets are shown in Fig. 7. It can be appreciated that there are similarities in waveform but the single-slot model is ignoring the effect of the neighbouring slots that is expressed mathematically as the boundary conditions on the middle of the teeth: (a) derivative of the radial component is zero and (b) the tangential component is zero. These two conditions are not satisfied by the waveform obtained with the single-slot model.

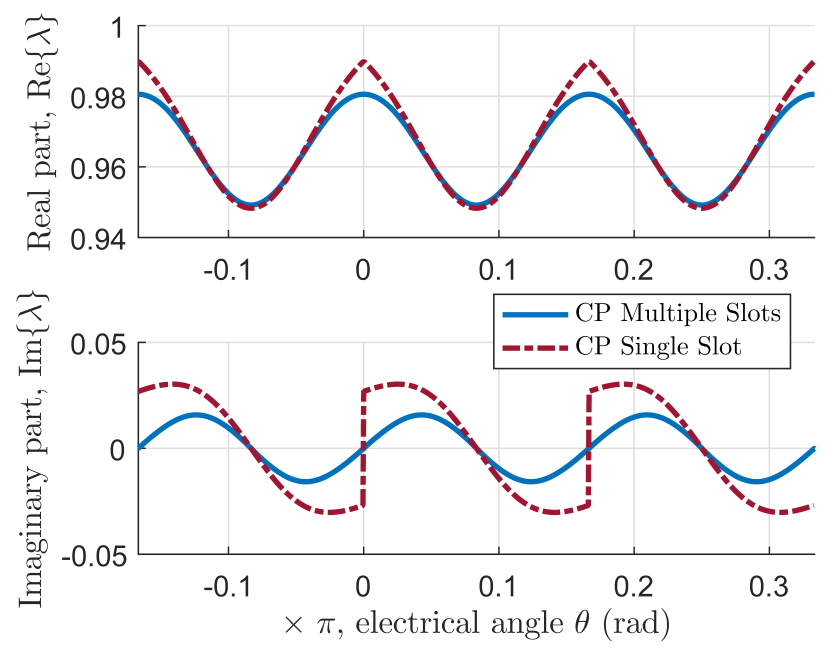

Fig. 7. Complex permeance function obtained with the multiple-slots and single-slot models; radial and tangential components.

Fig. 8 shows the waveform of the radial component of the no-load magnetic field distribution on the surface of the magnet at a particular rotor position using two-dimensional static FEA, multiple-slots model and single-slot model. It can be appreciated that the waveforms look very similar in Fig. 8. However, the amplitudes of the asynchronous harmonics have some significant differences because of the singularities and discontinuities of the single-slot CP function shown in Fig. 7. The amplitude of the most significant asynchronous harmonics is shown in Fig. 9. The singleslot model overestimates significantly the amplitude of the asynchronous harmonics.

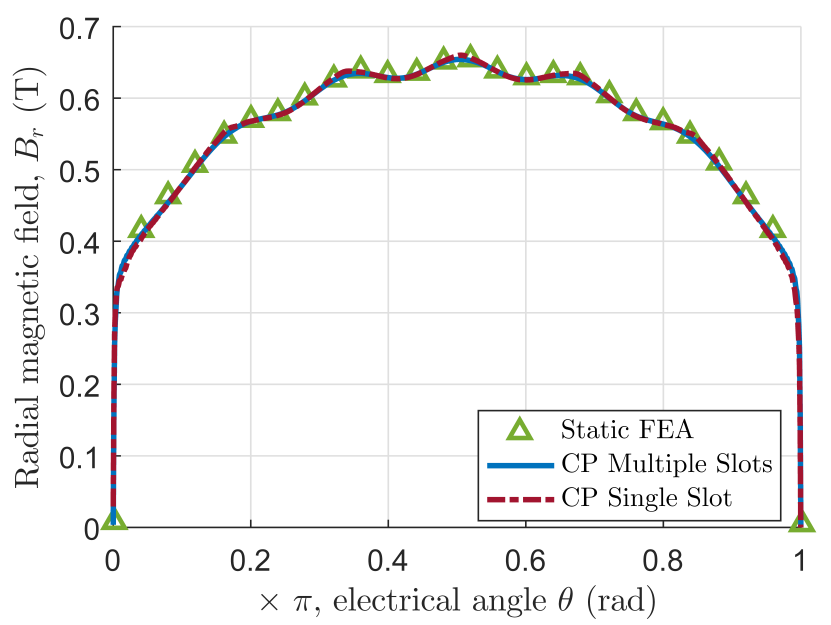

Fig. 8. Magnetic field distribution in the air-gap obtained using twodimensional static FEA and the CP function.

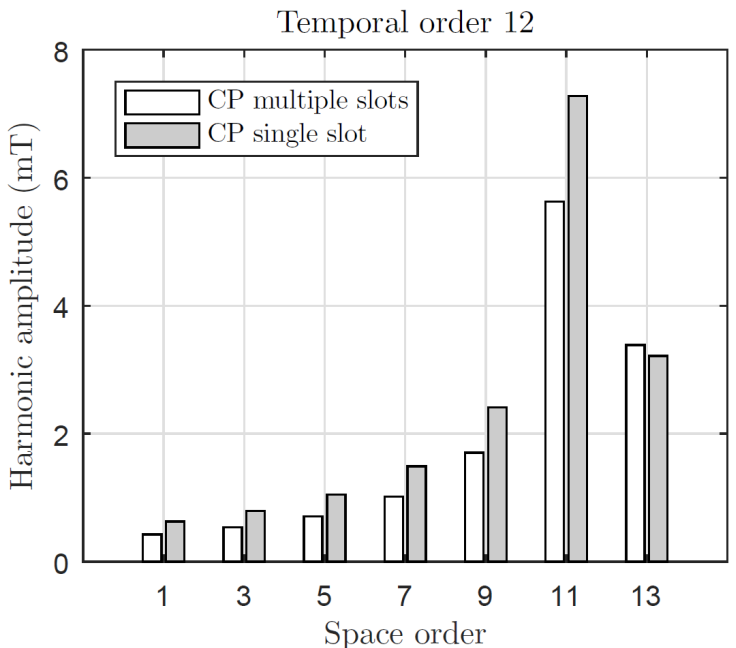

Fig. 9. No-load amplitude of the significant magnetic induction space harmonics of time order 12 at $90000 \mathrm{rpm}$.

Table II shows a comparison of the no-load losses obtained with the linear transient FEA calculations, the multiple-slots $\mathrm{CP}$ function and the single-slot $\mathrm{CP}$ function for the machine under study at running at $90000 \mathrm{rpm}$.

The rotor losses obtained with the single-slot $\mathrm{CP}$ function are significantly higher than the result obtained with FEA. On the other hand, the value obtained with the multiple-slots $\mathrm{CP}$ function has a good agreement (around $4 \mathrm{~W}$ error) compared to the single-slot model (around $18 \mathrm{~W}$ error) which could in 
TABLE II

NO-LOAD ROTOR POWER LOSS

\begin{tabular}{ll}
\hline Transient FEA & $\approx 11.2 \mathrm{~W}$ \\
Multiple-slots model & $\approx 15.5 \mathrm{~W}$ \\
Single-slot model & $\approx 29.4 \mathrm{~W}$ \\
\hline
\end{tabular}

some cases make the difference as far as the feasibility of a design variant.

The complex permeance function used in this paper assumes rectangular slots without tooth-tips, which is valid if there is not saturation in the tooth-tips as discussed in [4], [13].

\section{CONCLUSION}

This paper tackles the problem of deciding when is it reasonable to use the simple single-slot model and when is it necessary to use the more complicated multiple-slots model to calculate the magnetic field distribution in the air-gap and rotor losses of PM electric machines. The final criterion proposed in this paper is based on the fundamental theory of conformal mapping. The practical limit shows when it is possible to use the single-slot model without incurring significant errors and the numerical limit shows when the multiple-slots model fails to provide an answer. With the information of these two limits Fig. 5 presents a clear and reasonable criterion to choose the model required for each particular case.

The case study presented here of a high speed PM motor illustrates the importance of using the appropriate model. Ignoring the effect of the adjacent slots, i. e., using a singleslot model, produces a significant error in the calculation of rotor losses because the machine is clearly in the region of multiple-slots method in Fig. 5. Using the multiple-slots model improves the accuracy considerably.

This paper provides an insight about how choosing the wrong slotting model can produce errors in the estimation of the performance of the machine, particularly in the parameters that depend on the asynchronous harmonics like the rotor losses.

\section{REFERENCES}

[1] P. J. Lawrenson, P. Reece, and M. C. Ralph, "Tooth-ripple losses in solid poles," Electrical Engineers, Proceedings of the Institution of, vol. 113, pp. 657-662, April 1966.

[2] R. Stoll and J. Sykulski, "Modelling tooth ripple losses in the solid pole faces of synchronous machines," COMPEL - The international journal for computation and mathematics in electrical and electronic engineering, vol. 11, no. 1, pp. 105-108, 1992.

[3] A. A. Qazalbash, S. M. Sharkh, N. T. Irenji, R. G. Wills, and M. A. Abusara, "Rotor eddy current power loss in permanent magnet synchronous generators feeding uncontrolled rectifier loads," IEEE Transactions on Magnetics, vol. 50, pp. 1-9, June 2014.

[4] A. A. Qazalbash, S. M. Sharkh, N. T. Irenji, R. G. Wills, and M. A. Abusara, "Calculation of no-load rotor eddy-current power loss in pm synchronous machines," IEEE Transactions on Magnetics, vol. 50, pp. 1-8, Sept 2014.

[5] A. A. Qazalbash, S. M. Sharkh, N. T. Irenji, R. G. Wills, and M. A. Abusara, "Rotor eddy loss in high-speed permanent magnet synchronous generators," IET Electric Power Applications, vol. 9, no. 5 , pp. $370-376,2015$.
[6] O. Keysan, M. Mueller, A. McDonald, N. Hodgins, and J. Shek, "Designing the c-gen lightweight direct drive generator for wave and tidal energy," Renewable Power Generation, IET, vol. 6, pp. 161-170, May 2012.

[7] C. J. Ifedi, B. C. Mecrow, S. T. M. Brockway, G. S. Boast, G. J. Atkinson, and D. Kostic-Perovic, "Fault-tolerant in-wheel motor topologies for high-performance electric vehicles," IEEE Transactions on Industry Applications, vol. 49, pp. 1249-1257, May 2013.

[8] F. Carter, "Note on air-gap and interpolar induction," Journal of the Institution of Electrical Engineers, vol. 29, pp. 925-933, July 1900.

[9] T. A. Driscoll and L. N. Trefethen, Schwarz-Christoffel Mapping. Cambridge ; New York : Cambridge University Press, 2002., 2002.

[10] W. J. Gibbs, Conformal transformation in electrical Engineering. Chapman and Hall, 1958.

[11] E. M. Freeman, "The calculation of harmonics, due to slotting, in the flux-density waveform of a dynamo-electric machine," Proceedings of the IEE - Part C: Monographs, vol. 109, pp. 581-588, September 1962.

[12] Z. Q. Zhu and D. Howe, "Instantaneous magnetic field distribution in brushless permanent magnet dc motors. iii. effect of stator slotting," IEEE Transactions on Magnetics, vol. 29, pp. 143-151, Jan 1993.

[13] D. Zarko, D. Ban, and T. A. Lipo, "Analytical calculation of magnetic field distribution in the slotted air gap of a surface permanent-magnet motor using complex relative air-gap permeance," IEEE Transactions on Magnetics, vol. 42, pp. 1828-1837, July 2006.

[14] K. Boughrara, B. L. Chikouche, R. Ibtiouen, D. Zarko, and O. Touhami, "Analytical model of slotted air-gap surface mounted permanentmagnet synchronous motor with magnet bars magnetized in the shifting direction," IEEE Transactions on Magnetics, vol. 45, pp. 747-758, Feb 2009.

[15] H. B. Dwight, Tables of Integrals and Other Mathematical Data. Macmillan, 1947.

[16] R. Rabinovici, "Magnetic field analysis of permanent magnet motors," IEEE Transactions on Magnetics, vol. 32, no. 1, pp. 265-269, 1996.

[17] Z. Q. Zhu, D. Howe, and C. C. Chan, "Improved analytical model for predicting the magnetic field distribution in brushless permanentmagnet machines," IEEE Transactions on Magnetics, vol. 38, pp. 229 238, Jan 2002.

\section{BIOGRAPHIES}

Jaime Renedo Anglada was born in Madrid, Spain in 1989. He received the M.Sc. degree in electrical engineering from the Comillas Pontifical University, Madrid, Spain, in 2013. He is currently pursuing the Ph.D. degree with the Mechatronics Research Group at the University of Southampton, U.K. His main research topic is the analytic study of electric machines and drives.

From 2012 to 2013 he was an invited student at the Institute of Research in Technology (IIT), a research center of ICAI School of Engineering, which belongs to the Comillas Pontifical University, Madrid, Spain. The research topic was the modelling of synchronous generators for stability analysis.

Suleiman M. Sharkh received the B.Eng. and Ph.D. degrees in electrical engineering from the University of Southampton, Southampton, U.K., in 1990 and 1994, respectively.

$\mathrm{He}$ is Professor of Power Electronics, Machines and Drives, and head of the Mechatronics Research Group at the University of Southampton. He is also the Managing Director of HiT Systems Ltd. He has published over 40 papers in academic journals and conferences. His main research interests are in the area of control, electrical machine and power electronics with applications to electric vehicles.

Prof. Sharkh is a member of the IEEE, the IET and a Chartered Engineer. He was the 2008 winner of The Engineer Energy Innovation Award for his work on rim driven thrusters and marine turbine generators.

Arfakhshand Ali Qazalbash completed his Masters \& PhD degrees from University of Manchester and University of Southampton in 2004 and 2014. Currently he is working as an Electromagnetic design engineer at EMERSON Industrial Automation, where he works on electromagnetic problems related to PM machines. 